\title{
APPLICATION OF PARALLEL ALGORITHM APPROACH FOR PERFORMANCE OPTIMIZATION OF OIL PAINT IMAGE FILTER ALGORITHM
}

\author{
Siddhartha Mukherjee \\ Samsung R\&D Institute, India - Bangalore
}

\begin{abstract}
This paper gives a detailed study on the performance of image filter algorithm with various parameters applied on an image of RGB model. There are various popular image filters, which consumes large amount of computing resources for processing. Oil paint image filter is one of the very interesting filters, which is very performance hungry. Current research tries to find improvement in oil paint image filter algorithm by using parallel pattern library. With increasing kernel-size, the processing time of oil paint image filter algorithm increases exponentially. I have also observed in various blogs and forums, the questions for faster oil paint have been asked repeatedly.
\end{abstract}

\section{KEYWORDS}

Image Processing, Image Filters, Linear Image Filters, Colour Image Processing, Spatial Image Filter, Oil Paint algorithm, Parallel Pattern Library.

\section{INTRODUCTION}

This document provides an analytical study on the performance of Oil Paint Image Filter Algorithm. There are various popular linear image filters are available. One of the very popular and interesting image filters is Oil Paint image effect. This algorithm, being heavy in terms of processing it is investigated in this study.

There related studies are detailed in the Section 7.

\section{BACKGROUND}

Modern days, human beings have hands full of digital companions, e.g. Digital Camera, Smart Phones, Tablets and laptops so on. Most of these are having built-in camera, which are widely used compared to traditional cameras. The usage of these has started a new stream of applications, which include various categories e.g. image editing, image enhancement, camera extension application and so on. Almost all of these applications include applying different kinds of image filters.

Image filters are of different kinds, with respect their nature of processing or mathematical model. The execution time of any image filter is a very important aspect, which specifies whether the filter is acceptable for an application with respect to its acceptable execution performance in a given environment. E.g. The exaction time of any image filter, clearly specifies, whether the filter can be used for pre-processing or post-processing. Oil Paint is one of the very popular linear 
Signal \& Image Processing : An International Journal (SIPIJ) Vol.5, No.2, April 2014

image filters, which is very heavy in terms of execution time. The due course of this paper portrays the investigation of the performance of oil paint image filter.

\section{INVESTIGATION METHOD}

A simple Win32 windows application is developed for simulation to analyse different types of image filters. The purpose of this windows application is to accept different JPG image files as an input, and apply different kinds of image filters on to it. In the process of applying image filters the application keeps log of processing time. The overall operation of the application is explained here.

\subsection{Operational Overview}

The application is realised with two primary inputs: input jpg image files and configuration of image filter parameters. The application is designed with three major components: user interface (UI), jpeg image encoder-decoder and image filter algorithm.

The UI is developed using Microsoft's Win32 APIs. For this sub-module I have written approximately $300 \mathrm{LOC}$ in C.

The image encoder and decoder component is designed with WIC (Windows Imaging Component), provided by Microsoft and developed with approximately $100 \mathrm{LOC}$ in C.

The following flowchart diagram shows the operational overview of the test environment. During this process of testing, the processing time is logged and analysed for the study. The Oil paint image filter algorithm is developed using the approach as mentioned in reference [3]. This algorithm and its subsequent improvements are in developed with $\mathrm{C}$ language, in approximated 300 LOC.

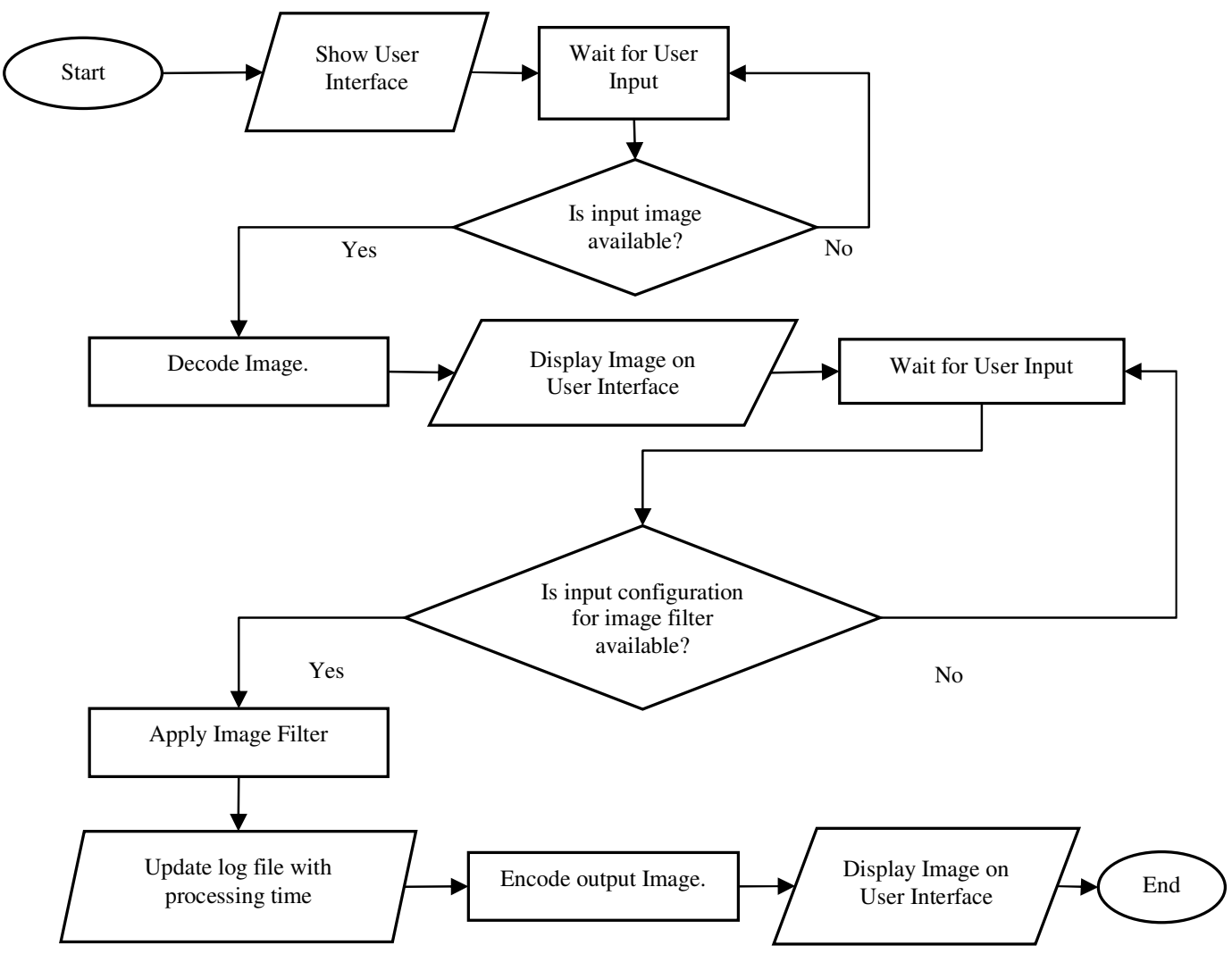


Signal \& Image Processing : An International Journal (SIPIJ) Vol.5, No.2, April 2014

\subsection{Implementation Overview}

Considering the above workflow diagram, main focus of the current study is done with the application of image filter (marked as "Apply Image Filter" operation). Other operations are considered to be well known and do not affect the study. The code snippet below will provide the clear view of implementation. The user interface can be designed in various ways; even this experiment can be performed without a GUI also. That is why the main operational point of interests can be realized with the following way.

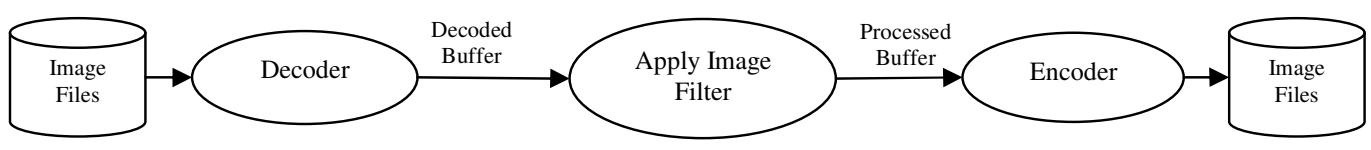

\section{Decoder}

The interface for decoding is exposed as shown here.

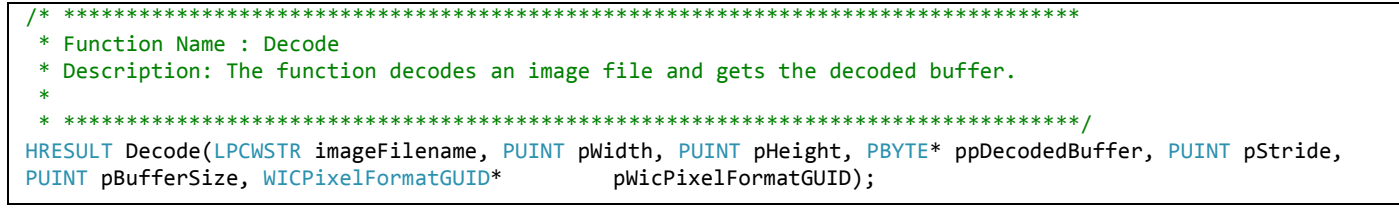

The implementation of the decoder interface is provided here.

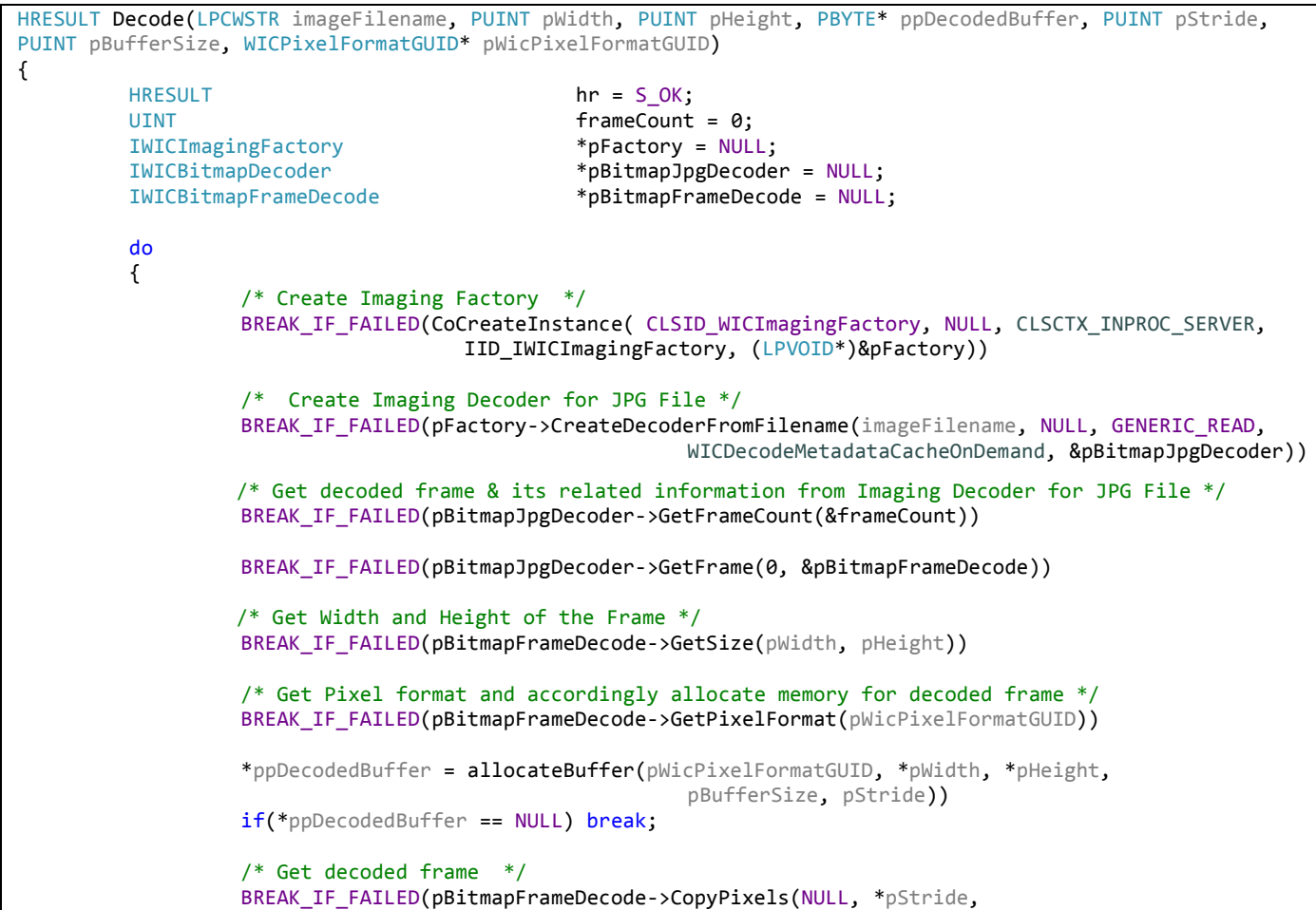


Signal \& Image Processing : An International Journal (SIPIJ) Vol.5, No.2, April 2014

\section{Encoder}

The interface for encoding is exposed as shown here.

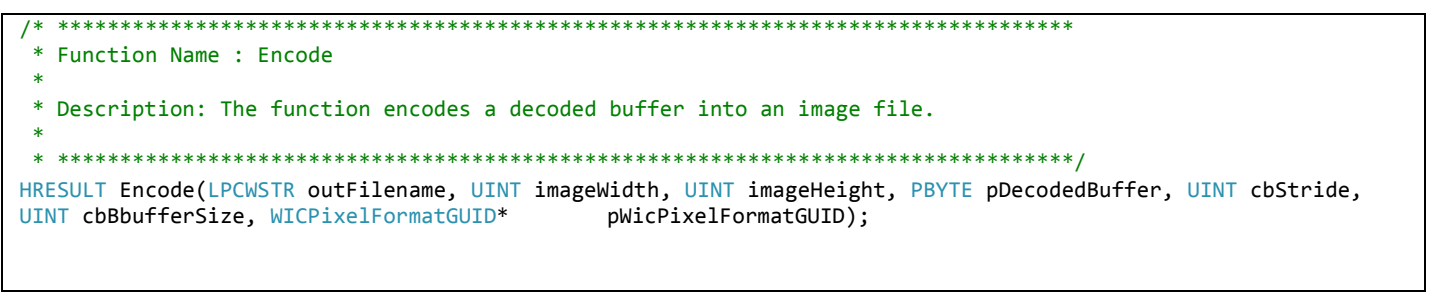

The implementation of the encoder interface is provided here.

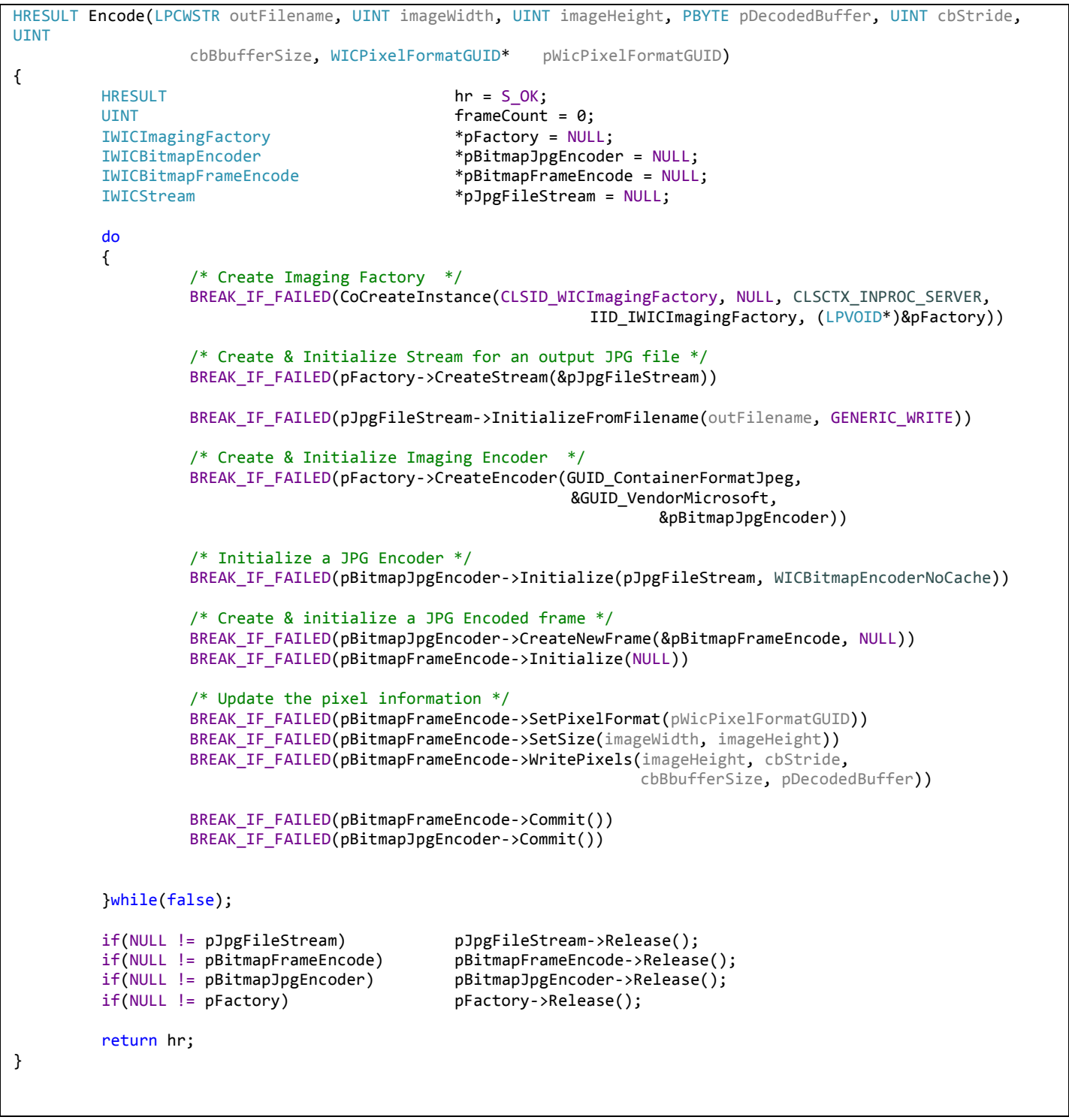


Signal \& Image Processing : An International Journal (SIPIJ) Vol.5, No.2, April 2014

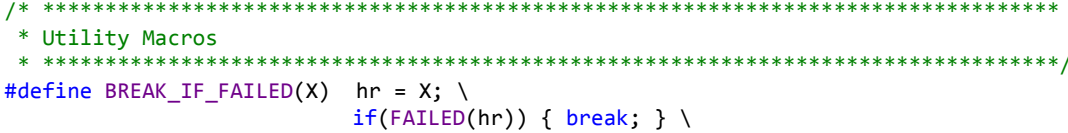

\section{Application of Image Filter}

The image processing algorithm is the subject of study in current experiment. Details of the algorithms are explained later. Following code snippet used to capture performances for any simple filter (e.g. oil paint).

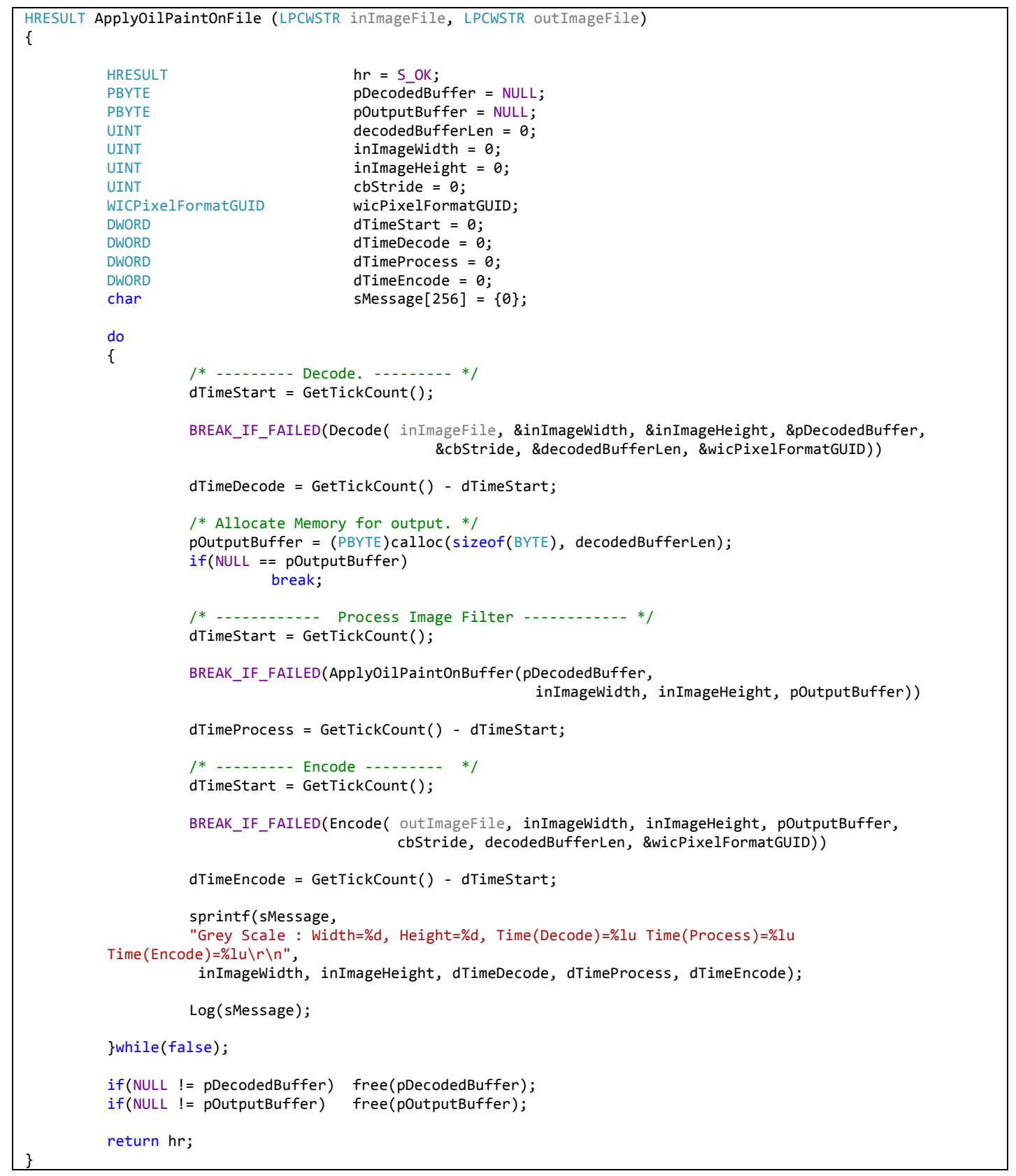


Signal \& Image Processing : An International Journal (SIPIJ) Vol.5, No.2, April 2014

For time measurement standard windows API GetTickCount is used. This API retrieves the number of milliseconds that have elapsed since the system was started.

\section{OIL Paint Image Filter in RGB COLOUR MOdel}

During this study, the input images are considered to be in RGB model. In this model, an image consists of two dimensional arrays of pixels. Each pixel of a 2D array contains data of red, green and blue colour channel respectively.

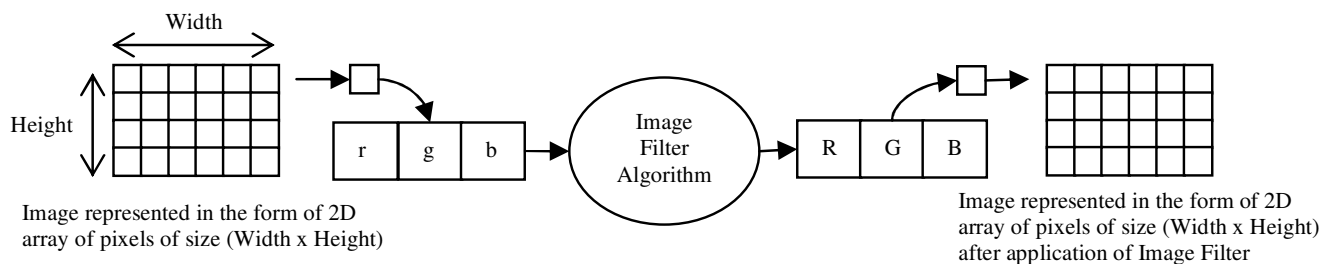

The Image Filters are basically algorithm for changing the values of Red, Green and Blue component of a pixel to a certain value. Depending upon the amount of access to neighbouring pixels in spatial domain, the performance of image filters is affected.

\section{Histogram based algorithm for Oil Paint}

This approach is mentioned in the reference [3]. For pixel at position $(\mathrm{x}, \mathrm{y})$, find the most frequently occurring intensity value in its neighbourhood. And set it as the new colour value at position $(\mathrm{x}, \mathrm{y})$.
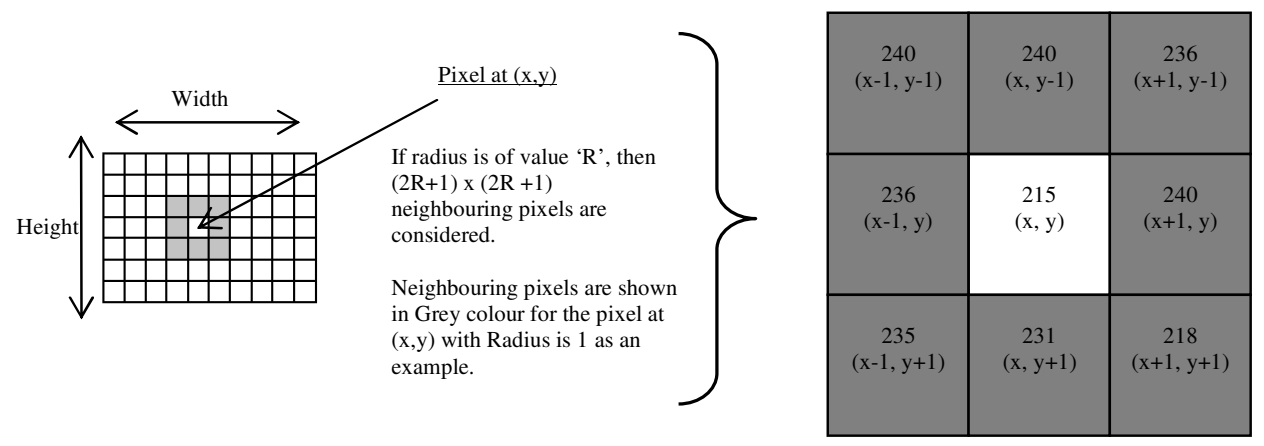

1) The right side provides the larger and clear picture of the neighbouring pixels or Radius 1, with respect to pixel at $(\mathrm{x}, \mathrm{y})$. The intensities of the respective pixels are also provided (as an example).

2) The pixels at $(x-1, y-1),(x, y-1),(x+1, y)$ have the maximum occurring intensity i.e. 240

3) The each colour channel of the pixel at $(x, y)$ is set with an average of each colour channel of 3 pixels $[(\mathrm{x}-1, \mathrm{y}-1),(\mathrm{x}, \mathrm{y}-1),(\mathrm{x}+1, \mathrm{y})]$

The intensity (I) of a pixel is calculated by equation $I=(R+G+B) / 3$. Here $R, G \& B$ are Red, Green and Blue component of a pixel.

The interface for the oil paint algorithm is exposed as follows.

* Function Name : ApplyOilPaintOnBuffer

* Description: Apply oil paint effect on decoded buffer.

$*$

HRESULT ApplyOilPaintOnBuffer(PBYTE pInBuffer, UINT width, UINT height, const UINT intensity_level, const int radius, PBYTE pOutBuffer);

Just mentioned oil paint algorithm is implemented as follows. 
Signal \& Image Processing : An International Journal (SIPIJ) Vol.5, No.2, April 2014

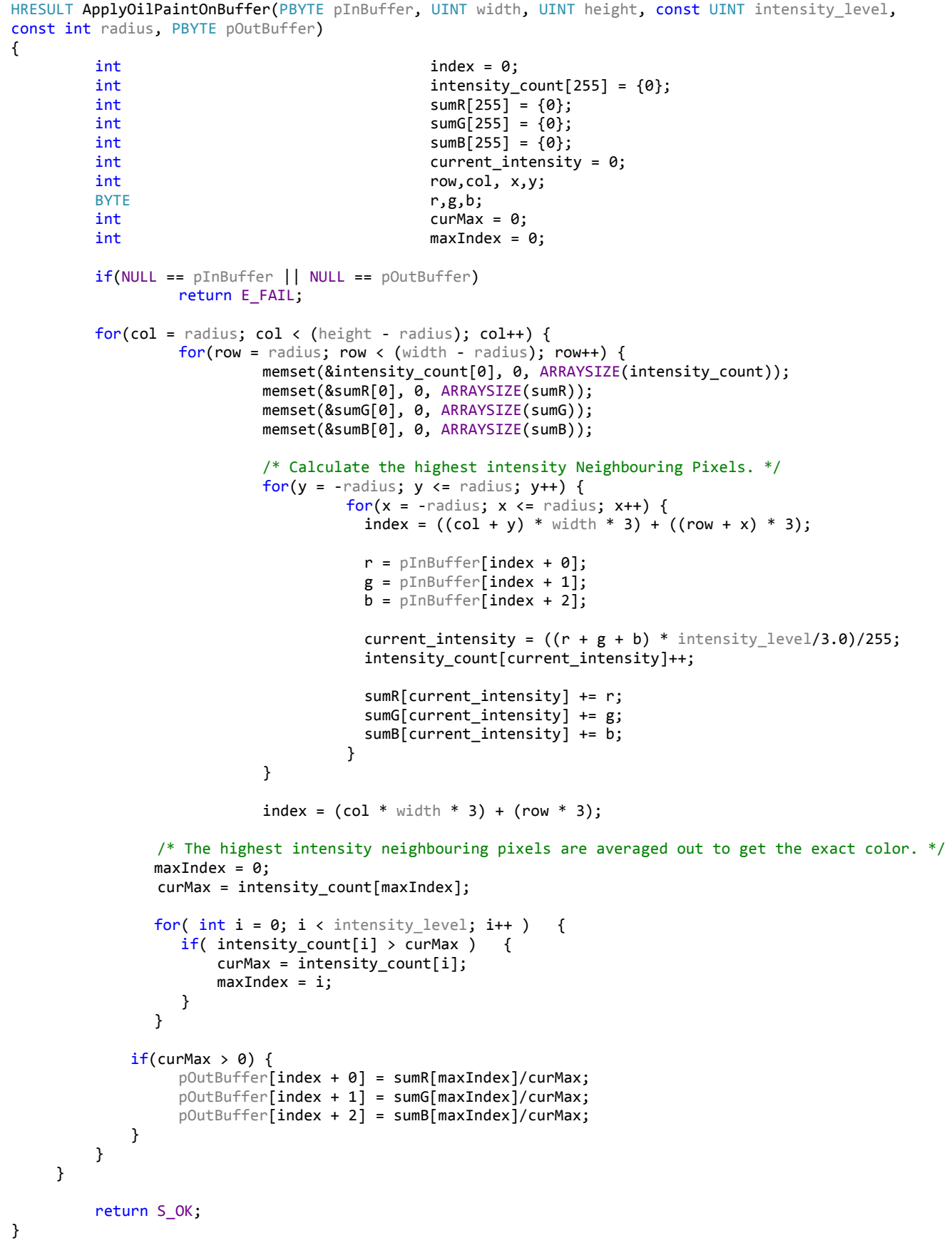

\section{Experimental Results}

The experimental is conducted with images of different size and application of oil paint with different radius. The following data shows the time of execution with different parameters. 
Signal \& Image Processing : An International Journal (SIPIJ) Vol.5, No.2, April 2014

\begin{tabular}{|c|c|c|}
\hline Size & Radius & Time \\
\hline $\operatorname{VGA}(640 \times 480)$ & 2 & 218 \\
\hline VGA(640x480) & 4 & 531 \\
\hline $\operatorname{VGA}(640 \times 480)$ & 6 & 1046 \\
\hline $\operatorname{VGA}(640 \mathrm{x} 480)$ & 8 & 1685 \\
\hline SVGA(800x600) & 2 & 297 \\
\hline SVGA(800x600) & 4 & 826 \\
\hline SVGA(800x600) & 6 & 1606 \\
\hline SVGA(800x600) & 8 & 2652 \\
\hline $\mathrm{XGA}(1024 \times 768)$ & 2 & 499 \\
\hline $\mathrm{XGA}(1024 \times 768)$ & 4 & 1326 \\
\hline $\mathrm{XGA}(1024 \times 768)$ & 6 & 2621 \\
\hline $\mathrm{XGA}(1024 \times 768)$ & 8 & 4383 \\
\hline FHD $(1920 \times 1080)$ & 2 & 1466 \\
\hline $\mathrm{FHD}(1920 \times 1080)$ & 4 & 3526 \\
\hline FHD(1920x1080) & 6 & 7020 \\
\hline FHD(1920x1080) & 8 & 11716 \\
\hline WQXGA(2560x1600) & 2 & 2559 \\
\hline WQXGA(2560x1600) & 4 & 6973 \\
\hline WQXGA(2560x1600) & 6 & 14008 \\
\hline WQXGA(2560x1600) & 8 & 23229 \\
\hline
\end{tabular}

\begin{tabular}{ll}
\hline System & Details \\
\hline Processor & Intel@ Core ${ }^{\mathrm{TM}}$ i7- \\
& 3630QM CPU @ 2.40 \\
& $\mathrm{GHz}, 2.40 \mathrm{GHz}$ \\
Operating & Windows 7 Enterprise, \\
System & $\begin{array}{l}64 \text { bit Operating } \\
\end{array}$ \\
RAM System. \\
\hline
\end{tabular}
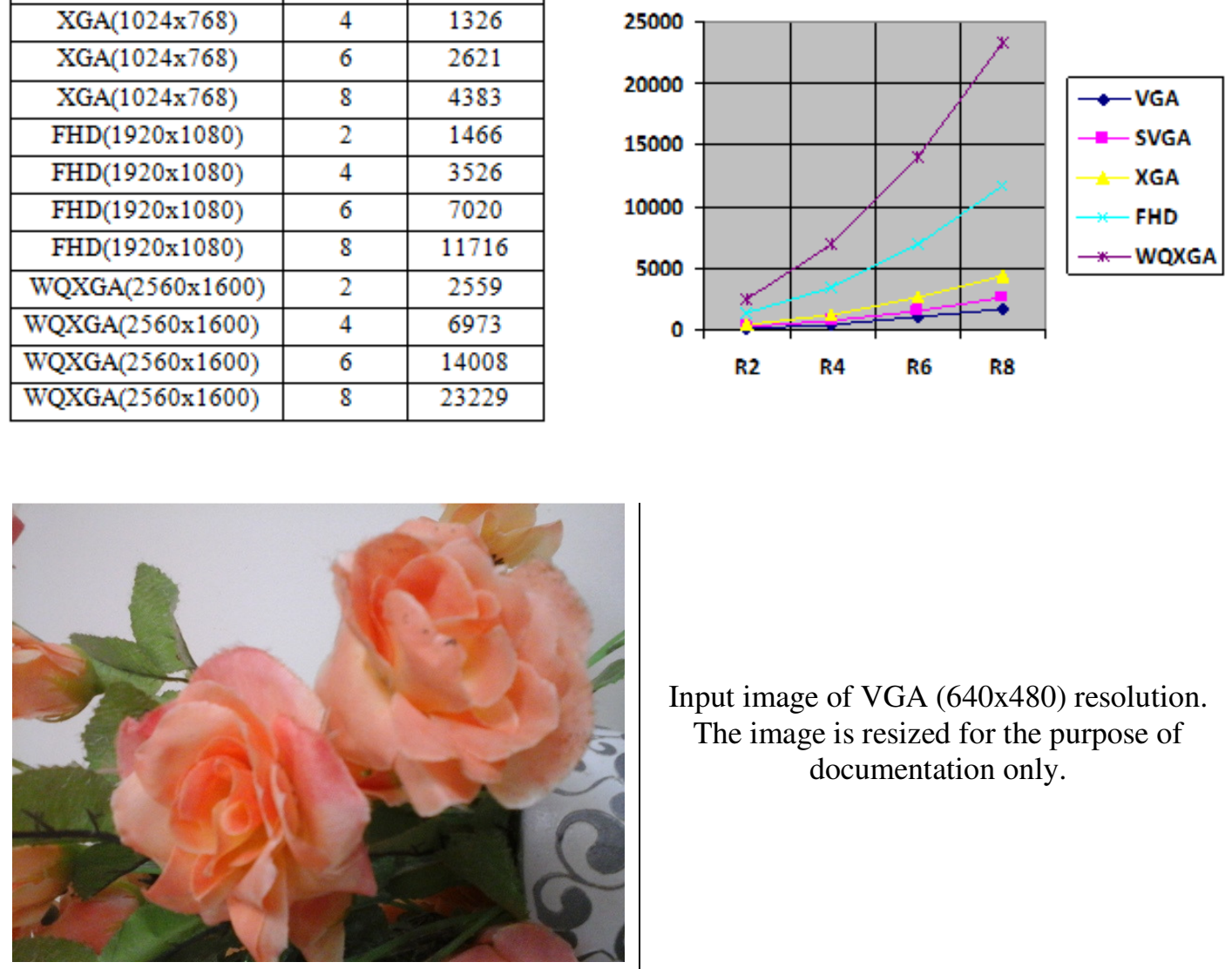

Input image of VGA $(640 \times 480)$ resolution.

The image is resized for the purpose of documentation only.

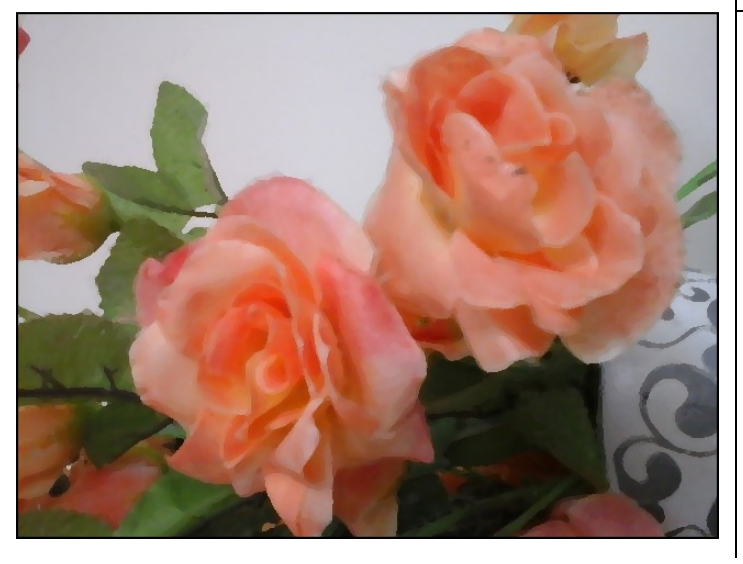

After application of oil paint algorithm with radius of 2. Output image resolution is VGA $640 \mathrm{x} 480$. The image is resized for the purpose of documentation only. 
Signal \& Image Processing : An International Journal (SIPIJ) Vol.5, No.2, April 2014

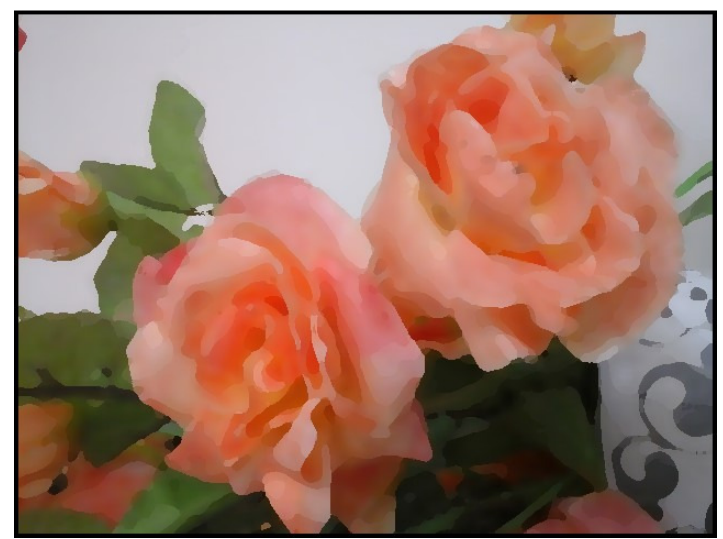

After application of oil paint algorithm with radius of 4. Output image resolution is VGA

640x480. The image is resized for the purpose of documentation only.

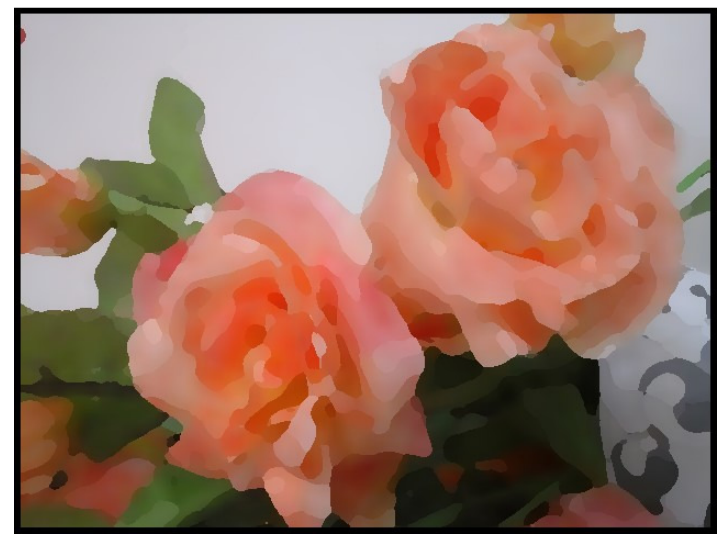

After application of oil paint algorithm with radius of 6 . Output image resolution is VGA $640 \times 480$. The image is resized for the purpose of documentation only.

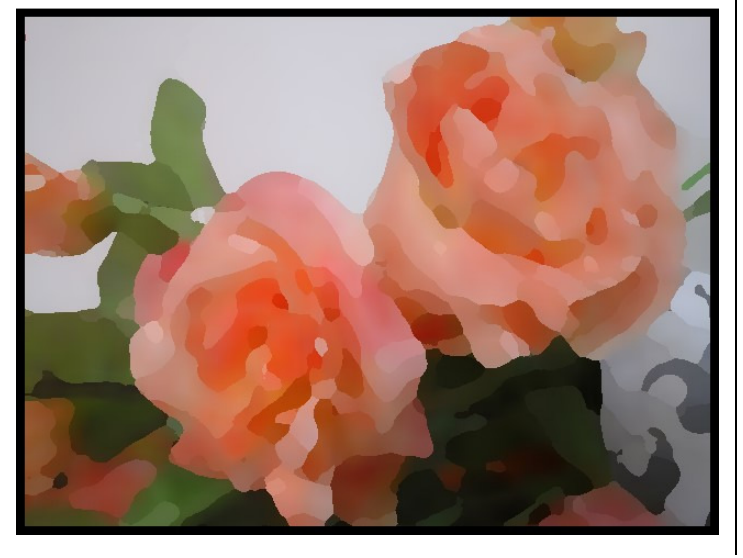

After application of oil paint algorithm with radius of 8 . Output image resolution is VGA $640 x 480$. The image is resized for the purpose of documentation only.

In due course of our investigation, I have observed that the performance of oil paint image filter increases in greater degree with increasing width, height and radius (i.e. usage of neighbouring pixel).

More importantly, I have observed most of the high resolution images are captured by more powerful camera. For these kinds of higher resolution photos, kernel size needs to be increased to generate Oil Paint effect of an acceptable quality 
Signal \& Image Processing : An International Journal (SIPIJ) Vol.5, No.2, April 2014

\section{Oil Paint Image Filter by Parallel Algorithm Approach}

I tried to improve the oil paint algorithm by Parallel Algorithm approach. I have used Microsoft Parallel Patterns Library for this purpose.

\section{Parallel Patterns Library}

The Parallel Patterns Library (PPL) is a Microsoft library designed for use by native C++ developers. PPL provides an imperative programming model that promotes scalability and easeof-use for developing concurrent applications. The PPL builds on the scheduling and resource management components of the Concurrency Runtime. It raises the level of abstraction between your application code and the underlying threading mechanism by providing generic, type-safe algorithms and containers that act on data in parallel.

The PPL provides the following features:

1) Task Parallelism: a mechanism to execute several work items (tasks) in parallel

2) Parallel algorithms: generic algorithms that act on collections of data in parallel

3) Parallel containers and objects: generic container types that provide safe concurrent access to their elements

Following code snippet will provide clear picture of the implementation using Microsoft PPL.

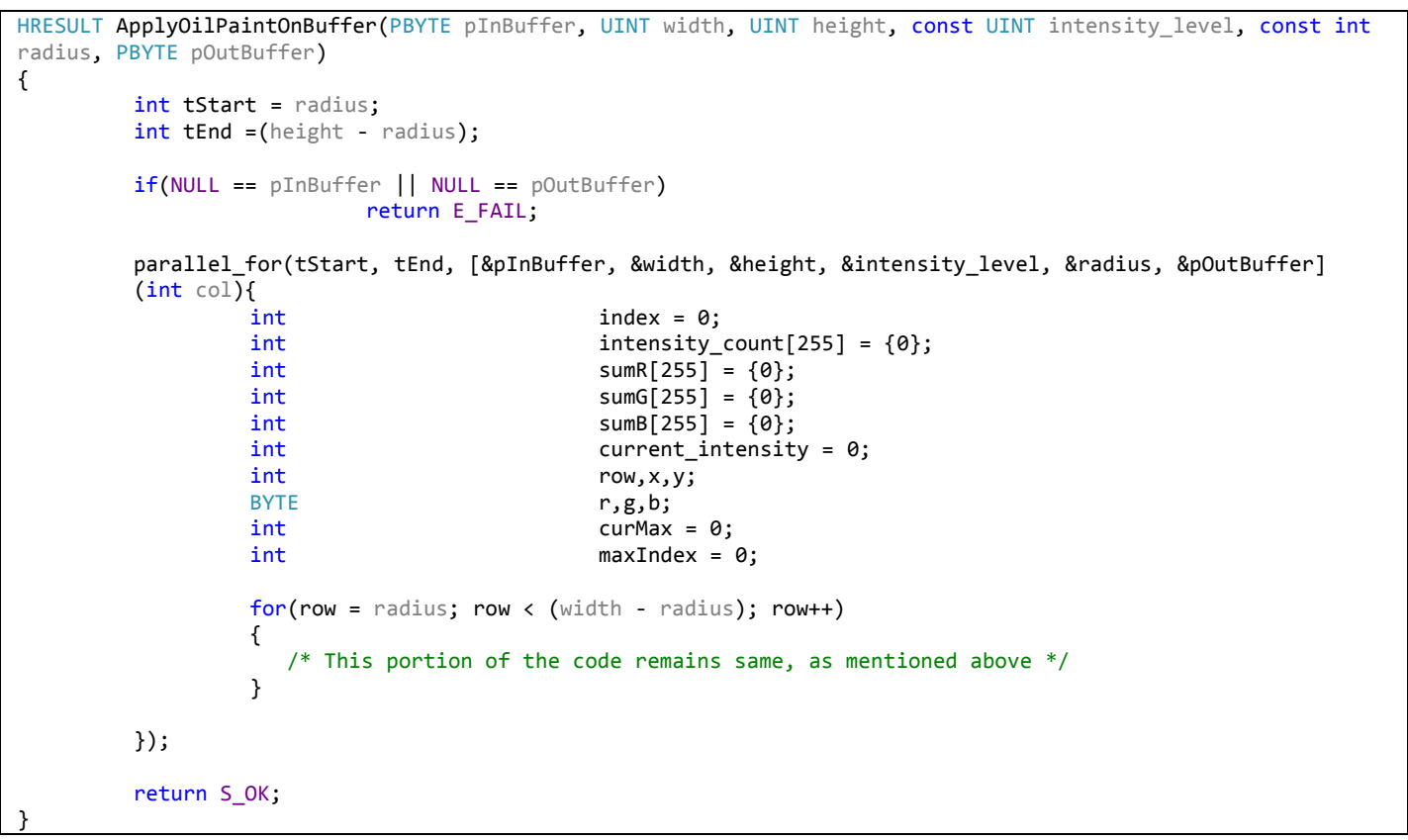

\section{Experimental Results}

The experiment is conducted with same set of images, used for the experiment, mentioned in the section above. Better execution performance is achieved with same quality, achieved in the previous stage of experiment. 
Signal \& Image Processing : An International Journal (SIPIJ) Vol.5, No.2, April 2014

\begin{tabular}{|c|c|c|}
\hline Size & Radius & Time \\
\hline VGA(640x480) & 2 & 94 \\
\hline $\mathrm{VGA}(640 \times 480)$ & 4 & 156 \\
\hline $\mathrm{VGA}(640 \times 480)$ & 6 & 281 \\
\hline VGA(640x480) & 8 & 483 \\
\hline SVGA( $(800 \times 600)$ & 2 & 78 \\
\hline SVGA( $(800 \times 600)$ & 4 & 234 \\
\hline SVGA( $(800 \times 600)$ & 6 & 452 \\
\hline SVGA( $(800 \times 600)$ & 8 & 734 \\
\hline $\mathrm{XGA}(1024 \times 768)$ & 2 & 140 \\
\hline $\mathrm{XGA}(1024 \times 768)$ & 4 & 375 \\
\hline $\mathrm{XGA}(1024 \times 768)$ & 6 & 733 \\
\hline $\mathrm{XGA}(1024 \times 768)$ & 8 & 1248 \\
\hline FHD $(1920 \times 1080)$ & 2 & 343 \\
\hline FHD $(1920 \times 1080)$ & 4 & 967 \\
\hline $\mathrm{FHD}(1920 \times 1080)$ & 6 & 1935 \\
\hline $\mathrm{FHD}(1920 \times 1080)$ & 8 & 3261 \\
\hline WQXGA(2560x1600) & 2 & 686 \\
\hline WQXGA(2560x1600) & 4 & 1872 \\
\hline WQXGA(2560x1600) & 6 & 3915 \\
\hline WQXGA(2560x1600) & 8 & 6490 \\
\hline
\end{tabular}

\begin{tabular}{ll}
\hline System & Details \\
\hline Processor & Intel@ Core ${ }^{\mathrm{TM}}$ i7- \\
& $3630 \mathrm{QMCPU}$ @ 2.40 \\
& $\mathrm{GHz}, 2.40 \mathrm{GHz}$ \\
Operating & Windows 7 Enterprise, \\
System & 64 bit Operating \\
& System. \\
RAM & $8.00 \mathrm{~GB}$ \\
\hline
\end{tabular}

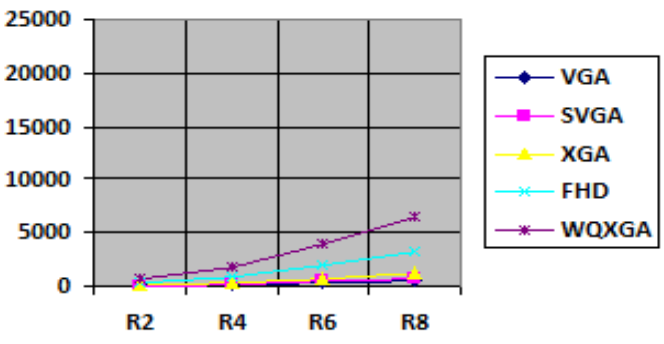

\section{COMPARATIVE ANALYSIS OF BOTH APPROACHES}

The improvement of the performance in terms of percentage is deduced as [100* (T1 - T2)/ t1], where $\mathrm{T} 1$ is time required for processing by $1^{\text {st }}$ approach and $\mathrm{T} 2$ is the time required for processing time by latest approach.

\begin{tabular}{|c|c|c|c|c|}
\hline Size & Radius & T1 & $\mathbf{T 2}$ & $\begin{array}{c}\text { Improvement } \\
(\%)\end{array}$ \\
\hline $\operatorname{VGA}(640 x 480)$ & 2 & 218 & 94 & 56.88073394 \\
\hline $\operatorname{VGA}(640 x 480)$ & 4 & 531 & 156 & 70.62146893 \\
\hline $\operatorname{VGA}(640 x 480)$ & 6 & 1046 & 281 & 73.13575526 \\
\hline $\operatorname{VGA}(640 \times 480)$ & 8 & 1685 & 483 & 71.33531157 \\
\hline $\operatorname{SVGA}(800 \times 600)$ & 2 & 297 & 78 & 73.73737374 \\
\hline $\operatorname{SVGA}(800 \times 600)$ & 4 & 826 & 234 & 71.67070218 \\
\hline SVGA(800x600) & 6 & 1606 & 452 & 71.85554172 \\
\hline SVGA $(800 \times 600)$ & 8 & 2652 & 734 & 72.32277526 \\
\hline XGA(1024x768) & 2 & 499 & 140 & 71.94388778 \\
\hline XGA(1024x768) & 4 & 1326 & 375 & 71.71945701 \\
\hline XGA(1024x768) & 6 & 2621 & 733 & 72.03357497 \\
\hline XGA(1024x768) & 8 & 4383 & 1248 & 71.52635181 \\
\hline FHD(1920x1080) & 2 & 1466 & 343 & 76.60300136 \\
\hline FHD(1920x1080) & 4 & 3526 & 967 & 72.57515598 \\
\hline FHD(1920x1080) & 6 & 7020 & 1935 & 72.43589744 \\
\hline FHD(1920x1080) & 8 & 11716 & 3261 & 72.16626835 \\
\hline WQXGA(2560x1600) & 2 & 2559 & 686 & 73.19265338 \\
\hline WQXGA(2560x1600) & 4 & 6973 & 1872 & 73.15359243 \\
\hline WQXGA(2560x1600) & 6 & 14008 & 3915 & 72.05168475 \\
\hline WQXGA(2560x1600) & 8 & 23229 & 6490 & 72.06078609 \\
\hline
\end{tabular}


CPU \& Thread Usage:

I have used 'Microsoft Resource Monitor' tool to analyse the CPU and Thread utilization of the system.

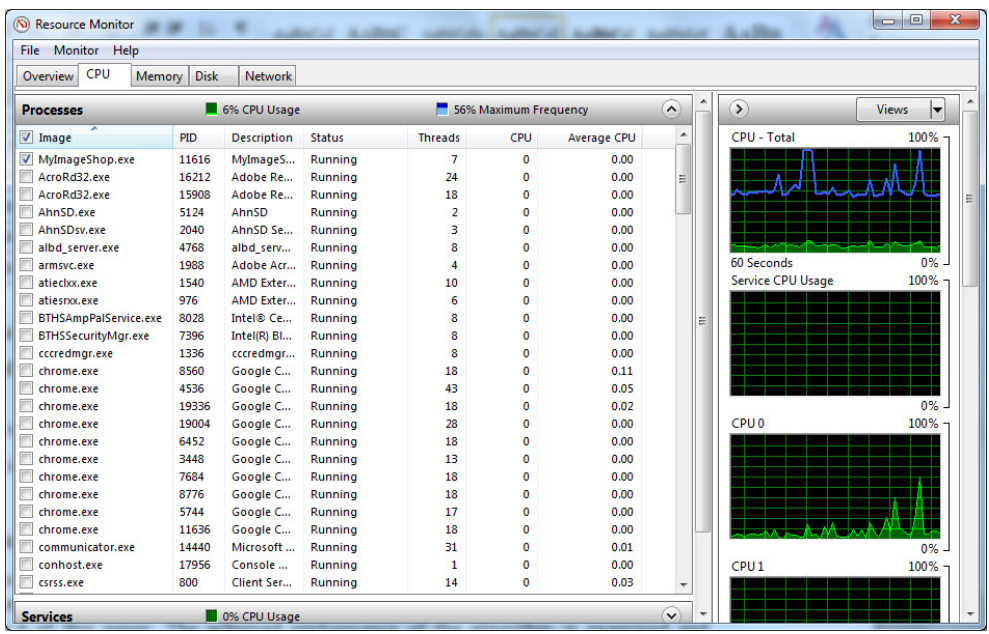

The application process is run and killed several times to find the average usage. This data is very likely to vary between various systems.

1) When PPL isn't used, minimum approximate CPU and Thread usage captured as $0.08 \& 2$

2) When PPL isn't used, maximum approximate CPU and Thread usage captured as $12.0 \& 20$

3) When PPL is used, minimum approximate CPU and Thread usage captured as $0.08 \& 8$

4) When PPL is used, maximum approximate CPU and Thread usage captured as $98 \& 36$

\section{REFERENCES STUDIED}

From reference [3] the histogram based oil paint image filter algorithm has been studied. The algorithm (mentioned in reference [3], section 'Oil-paint Effect') is implemented, as explained in the section 4 of this paper. The achieved performance of the algorithm is examined and captured in the section 4 (sub-section: Experimental Result) here. The result shows high growth of the processing time with respect to kernel-size. Reference [4] is another reference, where algorithm similar reference [3] is proposed for implementation. The reference [1] and [2] are used for way of analysis and follow the broadened scope in this arena of image processing. Reference [5] also proposes algorithm which are similar in nature with reference [3]. So we can clearly depict algorithms similar to reference [3] and [5], will face similar performance problem.

\section{Conclusions}

As in section $4 \& 7$, obtained result depicts huge growth in processing time with respect to the increase in kernel size. The current paper conducts study on improving execution time of oil paint image filter algorithm using the Microsoft technology.

As shown in section 6, I conclude Microsoft Parallel Pattern library yielded 71.6\% (average) performance improvement for Oil Paint Algorithm in given environment.

\section{Applicability:}

There are various similar image filter algorithm, where processing depends on neighbouring pixels. The image filters, face similar performance issues, as oil paint. The approach mentioned in this paper can be applied for similar issues. 
Signal \& Image Processing : An International Journal (SIPIJ) Vol.5, No.2, April 2014

In future, more well-known or new techniques in conjunction with the current idea can be used for betterment. Not only in image processing in other dimensions of signal processing as well similar approach can be tried.

\section{Limitations and Areas of improvement:}

The library for parallel execution depends on multi-core processing architecture. The result may differ on different processing architecture. More importantly, parallel pattern library may not be available for various operating systems (mainly, embedded devices). In those areas, the current approach may not be effective. Other programming techniques can be incorporated in those areas.

\section{ACKNOWLEDGEMENTS}

I thank my organization to provide me the opportunity for conducting this research!

\section{REFERENCES}

[1] Dr.G.Padmavathi, Dr.P.Subashini, Mr.M.Muthu Kumar and Suresh Kumar Thakur (2009) "Performance analysis of Non Linear Filtering Algorithms for underwater images", (IJCSIS) International Journal of Computer Science and Information Security. Vol.6, No. 2, 2009

[2] Aaron Hertzmann (1998) "Painterly rendering with curved brush strokes of multiple sizes", Proceedings of the 25th annual conference on Computer graphics and interactive techniques. Pages 453-460

[3] Feng Xiao (2000) “Oil-paint Effect”. Spring 2000/EE368 Course Project.

[4] Oil Paint Algorithm [http://supercomputingblog.com/graphics/oil-painting-algorithm/]

[5] P. S. Grover, Priti Sehgal (2004) “A Proposed Glass-Painting Filter". University of Delhi /icvgip/2004 / proceedings /cg1.1_109

[6] Parallel Patterns Library (PPL) [http://en.wikipedia.org/wiki/Parallel_Patterns_Library]

\section{Authors}

Siddhartha Mukherjee is a B.Tech (Computer Science and Engineering) from RCC Institute of Information Technology, Kolkata. Siddhartha is currently working as a Technical Manager in Samsung R\&D Institute, India- Bangalore. Siddhartha has almost 10 years of working experience in software development. He has previously worked with Wipro Technologies. He has been contributing for various technical papers \& innovations at different forums in Wipro and Samsung. His main area of work is mobile application developments.

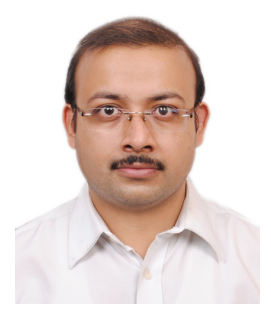

